\title{
Personalized Treatment for a Patient With a BRAF V600E Mutation Using Dabrafenib and a Tumor Treatment Fields Device in a High-Grade Glioma Arising From Ganglioglioma
}

\author{
Silviya K. Meletath, MD, MBS ${ }^{\mathrm{a}}$; Dean Pavlick ${ }^{\mathrm{b}}$; Tim Brennan, $\mathrm{MS}^{\mathrm{b}}$; Roy Hamilton, MD, MSc; \\ Juliann Chmielecki, $\mathrm{PhD}^{\mathrm{b}}$; Julia A. Elvin, $\mathrm{MD}, \mathrm{PhD}^{\mathrm{b}}$; Norma Palma ${ }^{\mathrm{b}}$ j Jeffrey S. Ross, $\mathrm{MD}^{\mathrm{b}, \mathrm{d}}$; \\ Vincent A. Miller, $\mathrm{MD}^{\mathrm{b}}$; Philip J. Stephens, $\mathrm{PhD}^{\mathrm{b}}$; George Snipes, $\mathrm{MD}^{\mathrm{a}}$; Veena Rajaram, MD; \\ Siraj M. Ali, MD, $\mathrm{PhD}^{\mathrm{b}}$; and Isaac Melguizo-Gavilanes, $\mathrm{MD}^{\mathrm{a}}$
}

\begin{abstract}
Background: Gangliogliomas are slow-growing, low-grade central nervous system tumors affecting children and young adults. However, some patients will experience tumor recurrence and/or malignant progression. This article reports on the clinical history, molecular findings, and treatment response in a patient with BRAF V600-mutated high-grade glioma arising from ganglioglioma. Methods: Hematoxylin-eosin staining and comprehensive genomic profiling via Foundation One were performed on the tumor sample from a male patient undergoing treatment at the Department of Neuro-Oncology at Baylor University Medical Center. Results: The patient was eligible for participation in a clinical trial (ClinicalTrials.gov identifier: NCT00916409) of a tumor treatment fields (TTFields) device, NovoTTF-100A, with concurrent radiation and chemotherapy (CCRT). His disease relapsed 4 months after completion of his CCRT, with MRI showing areas of enhancement. Temozolomide was discontinued and he was offered dabrafenib, an oral selective inhibitor of BRAF V600E, with continued use of NovoTTF. At the time of this report, after 2 years of treatment with dabrafenib and TTFields, the patient shows a durable complete response in all areas with no active lesions or new areas of enhancement. Conclusions: This report suggests that TTFields delivered in combination with targeted therapy dabrafenib yielded a remarkable clinical and radiologic response in this recurrent high-grade glioma. Targeted therapy matched to genomic alterations combined with TTFields treatment could provide clinical benefit and should be prospectively explored in the near future.
\end{abstract}

J Natl Compr Canc Netw 2016;14(11):1345-1350

\section{Background}

Gangliogliomas are rare central nervous system tumors of neuronal or mixed neuronal and glial origin capable of differentiating into either or both components. These tumors are usually located in the supratentorial region with temporal lobe predominance. ${ }^{1}$ They are classified as grade I tumors based on the current WHO classification system for central nervous system tumors. Anaplastic transformation of a ganglioglioma to a high-grade glioma (WHO

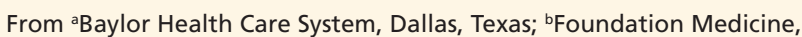
Inc., Cambridge, Massachusetts; 'Penn Memory Center, Department of Neurology, Philadelphia, Pennsylvania; ${ }^{d}$ Albany Medical College, Albany, New York; and eUT Southwestern Medical Center, Dallas, Texas.

Submitted December 30, 2015; accepted for publication July 12, 2016.

Drs. Pavlick, Brennan, Chmielecki, Palma, Ross, Miller, Stephens, and Ali are/ were employees of and have equity interest in Foundation Medicine, Inc. grade III) is rare and typically results in poor patient survival of 2 years or less. ${ }^{2}$ This article reports a case of malignant transformation of a ganglioglioma. Initial treatment with temozolomide and radiation was unsuccessful in achieving disease control, as was the combination of temozolomide and tumor treatment fields (TTFields) in the context of a clinical trial. Comprehensive genomic profiling (CGP) of this lesion identified a BRAF V600Eactivating mutation in the background of a near diploid 
Meletath et al

genome, and subsequent addition of dabrafenib and TTFields resulted in a durable complete response of more than 2 years.

\section{Case Presentation}

We reviewed the medical records of a male patient who is undergoing treatment at the Department of Neuro-Oncology at Baylor University Medical Center (Sammons Cancer Center). In accordance with the Baylor Institutional Review Board, the patient and his family provided informed consent to participate in an investigational TTFields trial, with NovoTTF-100A, for glioblastoma (GBM). Approval from the sponsor, patient, and family was obtained to review and submit the patient's deidentified clinical information and to report these data in the medical literature. Clinical and neurologic assessments were performed per protocol once every 4 to 5 weeks ${ }^{3}$ and brain MRIs were obtained every 8 weeks. Tumor response was determined using the Macdonald criteria. ${ }^{4}$

A 25-year-old man presented to our clinic in February 2013, after being diagnosed with a residual/ recurrent high-grade glioma thought to arise from a prior longstanding ganglioglioma. His original diagnosis of an intracranial lesion was made at Children's Medical Center, Dallas, in 1999. He had sustained a head injury while playing in a park and was incidentally found to have the lesion on a routine CT scan. He was monitored clinically for 3 years, but subsequently underwent subtotal resection of the tumor in February 2004, after it was observed to have increased in size in the left parietal periventricular region. The surgical pathology report noted features of ganglioglioma with mixed neuronal and glial growth patterns. In regions of the tumor with an abundant glial component, there was evidence of moderate cell proliferation as assessed by Ki-67 (MIB-1) immunohistochemistry (A, B). The patient did not receive any adjuvant chemotherapy or radiation at that time, and right-sided weakness after surgery resolved within a couple of months.

In February 2013, he experienced a sudden onset of right-sided hemiparesis and nonfluent aphasia. A noncontrast head CT and brain MRI revealed a left frontoparietal intracerebral hemorrhage and a left paraventricular mass. He underwent a left parietal craniotomy for evacuation of the hemorrhage, biopsy, and partial resection of the mass. Pathol- ogy showed high-grade glioma with characteristic features of GBM, but the finding of a BRAF V600E mutation by polymerase chain reaction and pyrosequencing (BRAF Pyro kit [Qiagen]) performed at med fusion (Lewisville, TX), and a previous diagnosis of ganglioglioma, was more consistent with an anaplastic ganglioglioma (WHO grade III). The IDH1 (R132H) mutation was not detected by immunohistochemistry. Features of tumor progression were indicated by a hypercellular glial component with relatively frequent mitotic figures, microvascular proliferation, and necrosis, with a tendency for pseudopalisading necrosis (Figure 1C-F). CGP was performed on the resected lesion. Methylguanine DNA methyltransferase (MGMT) testing was not obtained due to sample limitations.

Slides of tumor tissue were subsequently submitted for hybrid capture-based CGP to Foundation Medicine (Cambridge, MA). CGP was performed using the Illumina HiSeq 2000 on indexed, adaptorligated, hybridization-captured libraries for 4,561 exons of 287 cancer-related genes and 47 introns of 19 genes frequently involved in rearrangements. The tumor sample was evaluated for clinically relevant genomic alterations defined as base substitutions, short insertions and deletions, amplifications, homozygous deletions, and chromosomal rearrangements (detected by chimeric pair analysis for DNA data using custom bait-sets). The genetic alterations evaluated are either associated with anticancer therapies currently on the market, or which could be used to direct patients toward registered clinical trials. Both publically available and validated analysis tools were used to analyze the data, which yielded a median exon coverage of $647 \mathrm{X}$ and $100 \%$ of target DNA sequenced at $100 \mathrm{X}^{5}$ or greater. CGP detected a BRAF V600E mutation and homozygous losses in CDKN2A, CDKN2B, and $B C O R$, in an otherwise diploid tumor genome.

The patient was initially treated with concurrent chemoradiation therapy (CCRT) that included initial loading with temozolomide concurrently with conformal radiation therapy $(5,940 \mathrm{cGy})$ followed by monthly temozolomide. He was eligible for the clinical trial "A Prospective, Multi-center Trial of NovoTTF100A Together With Temozolomide Compared to Temozolomide Alone in Patients with Newly Diagnosed GBM" (ClinicalTrials.gov identifier: NCT00916409), given stable MRI post-CCRT (Figure 2A,B), consented to enrollment, and was randomized to the combi- 




Figure 1. (A, B) Light micrographs of the original ganglioglioma (original magnification $\mathrm{x} 200$ ). (A) Tissue stained with hematoxylin-eosin (H\&E) showing low-grade glioma; the inset shows immunohistochemical staining for glial fibrillary acidic protein. (B) Neuronal nuclei (NeuN) immunohistochemistry demonstrating an abnormal proliferation of neoplastic neurons. (C-F) Light micrographs of the recurrent high-grade ganglioglioma (original magnification $\times 200$ ). (C) A region consistent with low-grade glioma with pleomorphic nuclei and a few Rosenthal fibers; the inset shows an eosinophilic granular body. (D) A moderate proliferation index by immunohistochemical staining for Ki-67. H\&E-stained sections of the higher-grade regions showing (E) microvascular proliferation and (F) pseudopalisading necrosis.

nation arm. However, he experienced disease relapse 4 months after completion of his CCRT, with an MRI revealing new areas of enhancement superior and medial to the left parietal lobe surgical cavity. Advanced DSC (dynamic susceptibility-weighted contrastenhanced) MRI imaging showed elevated relative cerebral blood volume up to 4.2:1 compared with the contralateral white matter, supporting hyperperfusion and tumor recurrence (Figure $2 \mathrm{C}-\mathrm{F}$ ).

Given the failure of the temozolomide-based trial regimen, temozolomide was discontinued and the patient was offered dabrafenib (150 mg twice daily), an oral selective inhibitor of BRAF V600E, with continued use of the TTFields device based on his genomic profile results. He developed a febrile drug reaction to dabrafenib 3.5 weeks after the start of treatment. Dabrafenib was transiently interrupted for 32 days, and he was treated symptomatically; he later resumed treatment at a decreased dose of $75 \mathrm{mg}$ in the morning and $150 \mathrm{mg}$ in the evening.

A follow-up MRI obtained 2 months after resuming dabrafenib showed a complete response with resolution of the new lesions (Figure $2 \mathrm{G}, \mathrm{H}$ ). At the time of this report, after 2 full years of treatment with dabrafenib and the TTFields device, the patient's most recent MRI continues to show a complete and durable response without active lesions or new areas of enhancement. Clinically, the patient's residual 



Figure 2. $(A, B)$ Conventional axial T1-weighted MRI with contrast at baseline post-chemoradiation, (C-F) at progression with corresponding advanced dynamic susceptibility-weighted contrastenhanced MRI imaging, and $(G, H)$ after addition of dabrafenib.

right-sided hemiparesis and nonfluent aphasia have stabilized. He has not experienced any other treatment-related adverse events. He wears the TTFields device continuously with greater than $90 \%$ compliance $^{3}$ and has required no antiepileptic drugs or steroids during his course of treatment.

\section{Discussion}

Gangliogliomas are slow-growing, low-grade central nervous system tumors affecting children and young adults. However, some patients will experience tumor recurrence and/or malignant progression. The transformation of a subset of pediatric low-grade gangliogliomas to high-grade anaplastic gangliogliomas correlates with a transition from an indolent to an aggressive clinical course, presenting management challenges. ${ }^{6-8}$ A retrospective review of anaplastic ganglioglioma cases from the SEER database reported a median overall survival of 28.5 months in patients with anaplastic gangliomas. ${ }^{2}$ The median time to progression for patients with high-grade tumors at diagnosis was 4.4 years and the 2-year progression-free survival for high-grade gangliogliomas was $25 \% .{ }^{9} \mathrm{Nev}$ ertheless, recurrence rates of anaplastic ganglioglioma range from $38 \%$ to $60 \%,{ }^{10,11}$ with $45 \%$ of those being secondary GBMs after repeat surgery. ${ }^{11}$ Our patient did not have a third surgery, and because malignant transformation in gangliogliomas is an uncommon event, there is controversy as to how these should ultimately be classified. His tumor was considered an anaplastic ganglioglioma; with the caveat of having another tumor recurrence at 4 months, one has to assume a more aggressive clinical course. The WHO classification considers anaplastic gangliogliomas as grade III tumors, noting the rarity of the tumor and the wide variation in outcomes associated with anaplastic histology, but the rapid recurrence in our patient, taken together with other reports in the literature, is more compatible with the clinical behavior of a grade IV glioma (GBM). ${ }^{6-8}$ In a small series of patients with malignant gangliogliomas, all patients with histologically proven or presumed secondary GBMs died from their disease. ${ }^{11}$ Furthermore, patients with recurrent GBM have objective response rates less than $7 \%, 12,13$ with a 6 -month progression-free survival of $9 \%$ and 12 -month overall survival of $14 \% .^{14}$

Of note, patients with high-grade glioma treated with radiation and chemotherapy can have pseudoprogression $^{15}$ defined by radiographic changes, such as increase in size of existing lesions or appearance of new areas of contrast enhancement on MRI studies, soon after treatments, with subsequent improvement without any further specific treatment.

In our patient, advanced imaging and timing of these lesions beyond 12 weeks from completion of CCRT favored true progression, because one does 
not want to exclude or delay fastest recurring tumors from necessary therapy changes; however, pseudoprogression cannot be ruled out entirely.

Given that we did not have data on MGMT promoter methylation, the relevance of this correlate with pseudoprogression could not be analyzed. It has been reported, however, that secondary GBMs may have a very low incidence of pseudoprogression, even in the presence of methylated MGMT status. ${ }^{16}$

BRAF alterations are common in gangliogliomas and include both missense mutations, such as V600E, and fusions that result in constitutively active kinases. ${ }^{17}$ BRAF V600E, activates MEK-ERK signaling $^{18}$ and is sensitive to BRAF V600E mutant-specific inhibitors such as vemurafenib and dabrafenib. 19,20 BRAF V600E mutations are also found in other lowgrade pediatric gliomas, such as pleomorphic xanthoastrocytomas and pilocytic astrocytomas with anaplasia (WHO grade II), as well as the higher-grade gliomas and anaplastic gangliogliomas (WHO grade III). ${ }^{21}$

In a recent study, BRAF V600E mutations and CDKN2A deletions were associated with posttransformation secondary high-grade gliomas. The presence of BRAF V600E distinguished secondary highgrade gliomas from primary high-grade gliomas, and BRAF and CDKN2A alterations were significantly less common in low-grade gliomas that did not transform. Pediatric low-grade gliomas with BRAF V600E mutations had a longer latency to transformation and higher 5-year overall survival versus gliomas harboring other oncogenic drivers and wild-type BRAF. ${ }^{22}$ Although the significance of and ability to target the BRAF alteration is striking, the relevance of the CDKN2A deletion is less so; although identified, it is doubtful that CDKN2A is a comparable oncogenic driver mutation, and may not impact patient response in this case.

Several reports describe cases of gliomas harboring BRAF V600E that have responded to targeted therapy. A pediatric case of recurrent right frontoparietal GBM, which harbored a BRAF V600E mutation, yielded a complete response after 4 months of vemurafenib therapy and remained sustained at 6 months. ${ }^{23}$ A case of a cervicomedullary ganglioglioma had a 12 -week continued overall decrease in BRAF V600E-mutated lesion size due to the combination of vemurafenib and the vinca alkaloid vinblastine. ${ }^{24} \mathrm{~A}$ recent report on a recurrent ganglioglioma in the left temporal lobe and posterior brain stem treated with dabrafenib showed significant clinical and radiographic response in an adult patient. ${ }^{25}$

Our report suggests superiority of targeted therapy matched to genomic alterations relative to chemotherapy, because the patient did not experience response to temozolomide but did experience response to dabrafenib while being treated with TTFields. We cannot conclude whether the response observed in this patient is from dabrafenib or from the combination of dabrafenib and TTFields. However, the dramatic and durable response evidenced with this combination relative to previous treatments supports possible synergies of targeted therapy and TTFields, as well as potentially novel mechanisms to counteract resistance to BRAF inhibitors. ${ }^{26,27}$ The only available data published to date with TTFields and targeted therapy is with bevacizumab in relapsed high-grade gliomas, ${ }^{28}$ showing a median overall survival of 7.9 weeks with no significant toxicity.

The TTFields device, Optune (Novocure, Portsmouth, $\mathrm{NH}$ ), is a portable battery-operated or power supply-operated device approved for the treatment of newly diagnosed and recurrent GBM. ${ }^{3,29}$ The system produces alternating electrical fields, TTFields, within the human body, which are applied to the head of the patient by electrically insulated surface transducer arrays. TTFields are believed to disrupt the rapid cell division exhibited by cancer cells. ${ }^{3,29}$ An improved quality of life has been reported in patients with GBM treated with TTFields compared with those with recurrent GBM treated with best standard of care. ${ }^{29}$ The clinical trial that our patient participated in (TTFields in combination with standard-of-care temozolomide) extended both progression-free and overall survival compared with temozolomide alone in patients with newly diagnosed GBM (ClinicalTrials.gov identifier: NCT00916409). Two-year survival increased from 29\% with temozolomide alone to $43 \%$ with TTFields in combination with temozolomide; progression-free survival increased from a median of 4.2 to 7.1 months; and overall survival increased from a median of 16.6 to 19.4 months. ${ }^{30}$ Additionally, post hoc analysis of this trial suggested that patients at first recurrence lived longer with the combination of TTFields and chemotherapy. ${ }^{31}$ 
Meletath et al

\section{Conclusions}

TTFields in combination with temozolomide did not display significant patient benefit in this case; however, TTFields delivered in combination with targeted therapy dabrafenib yielded a remarkable clinical and durable complete radiologic response. The positive response seen in this patient raises the possibility that this novel combination may have the potential to provide a sustained response for other patients with glioma also harboring BRAF V600E and CDKN2A loss. For this rare variant of malignant glioma, targeted therapy matched to genomic alterations combined with TTFields treatment could provide large clinical benefit and should be prospectively explored in the near future.

\section{References}

1. Matsuzaki K, Uno M, Kageji T, et al. Anaplastic ganglioglioma of the cerebellopontine angle. Case report. Neurol Med Chir (Tokyo) 2005;45:591-595.

2. Selvanathan SK, Hammouche S, Salminen HJ, Jenkinson MD. Outcome and prognostic features in anaplastic ganglioglioma: analysis of cases from the SEER database. J of Neurooncol 2011;105:539-545

3. U. S. Food and Drug Administration Neurological Devices Panel. March 17, 2011. NovoCure Ltd. NovoTTF-100A System. PMA P100034. Available at: http://www.fda.gov/downloads/ AdvisoryCommittees/CommitteesMeetingMaterials/MedicalDevices/ MedicalDevicesAdvisoryCommittee/NeurologicalDevicesPanel/ UCM247168.pdf. Accessed October 10, 2016.

4. Macdonald DR, Cascino TL, Schold SC Jr, Cairncross JG. Response criteria for phase II studies of supratentorial malignant glioma. J Clin Oncol 1990;8:1277-1280.

5. Frampton GM, Fichtenholtz A, Otto GA, et al. Development and validation of a clinical cancer genomic profiling test based on massively parallel DNA sequencing. Nat Biotechnol 2013;31:1023-1031.

6. Lee CC, Wang WH, Lin CF, et al. Malignant transformation of supratentorial ganglioglioma. Clin Neurol Neurosurg 2012;114:13381342.

7. Mittelbronn M, Schittenhelm J, Lemke D, et al. Low grade ganglioglioma rapidly progressing to a WHO grade IV tumor showing malignant transformation in both astroglial and neuronal cell components. Neuropathology 2007;27:463-467.

8. Rumana CS, Valadka AB. Radiation therapy and malignant degeneration of benign supratentorial gangliogliomas. Neurosurgery 1998;42:10381043.

9. Yust-Katz S, Anderson MD, Liu D, et al. Clinical and prognostic features of adult patients with gangliogliomas. Neuro Oncol 2014;16:409-413.

10. Blumcke I, Wiestler OD. Gangliogliomas: an intriguing tumor entity associated with focal epilepsies. J Neuropathol Exp Neurol 2002;61:575584.

11. Majores $\mathrm{M}$, von Lehe $\mathrm{M}$, Fassunke J, et al. Tumor recurrence and malignant progression of gangliogliomas. Cancer 2008;113:3355-3363.
12. Wick W, Puduvalli VK, Chamberlain MC, et al. Phase III study of enzastaurin compared withlomustine in the treatment of recurrent intracranial glioblastoma. J Clin Oncol 2010;28:1168-1174.

13. Yung WK, Albright RE, Olson J, et al. A phase II study of temozolomide vs. procarbazine in patients with glioblastoma multiforme at first relapse. Br J Cancer 2000;83:588-593.

14. Ballman KV, Buckner JC, Brown PD, et al. The relationship between sixmonth progression-free survival and 12 -month overall survival end points for phase II trials in patients with glioblastoma multiforme. Neuro Oncol 2007;9:29-38.

15. Melguizo-Gavilanes I, Bruner JM, Guha-Thakurta N, et al. Characterization of pseudoprogression in patients with glioblastoma: is histology the gold standard? J Neurooncol 2015;123:141-150.

16. Juratli TA, Engellandt $K$, Lautenschlaeger I, et al. Is there pseudoprogression in secondary glioblastomas? Int J Radiat Oncol Biol Phys 2013;87:10941099.

17. Appin CL, Brat DJ. Molecular pathways in gliomagenesis and their relevance to neuropathologic diagnosis. Adv Anat Pathol 2015;22:50-58.

18. Holderfield M, Deuker MM, McCormick F, McMahon M. Targeting RAF kinases for cancer therapy: BRAF-mutated melanoma and beyond. Nat Rev Cancer 2014;14:455-467.

19. McArthur GA, Chapman PB, Robert C, et al. Safety and efficacy of vemurafenib in $\mathrm{BRAF}(\mathrm{V} 600 \mathrm{E})$ and $\mathrm{BRAF}(\mathrm{V} 600 \mathrm{~K})$ mutation-positive melanoma (BRIM-3): extended follow-up of a phase 3, randomised, openlabel study. Lancet Oncol 2014;15:323-332.

20. Hauschild A, Grob JJ, Demidov LV, et al. Dabrafenib in BRAF-mutated metastatic melanoma: a multicentre, open-label, phase 3 randomised controlled trial. Lancet 2012;380:358-365.

21. Schindler G, Capper D, Meyer J, et al. Analysis of BRAF V600E mutation in 1,320 nervous system tumors reveals high mutation frequencies in pleomorphic xanthoastrocytoma, ganglioglioma and extra-cerebellar pilocytic astrocytoma. Acta Neuropathol 2011;121:397-405.

22. Mistry M, Zhukova N, Merico D, et al. BRAF mutation and CDKN2A deletion define a clinically distinct subgroup of childhood secondary high grade glioma. J Clin Oncol 2015;33:1015-1022.

23. Robinson GW, Orr BA, Gajjar A. Complete clinical regression of a BRAF V600E-mutant pediatric glioblastoma multiforme after BRAF inhibitor therapy. BMC Cancer 2014;14:258.

24. Rush S, Foreman N, Liu A. Brainstem ganglioglioma successfully treated with vemurafenib. J Clin Oncol 2013;31:e159-160.

25. Shih KC, Shastry M, Williams JT, et al. Successful treatment with Dabrafenib (GSK 2118436) in a patient with ganglioglioma. J Clin Oncol 2014;32:e98-100.

26. Hartsough E, Shao $\mathrm{Y}$, Aplin AE, et al. Resistance to RAF inhibitors revisited. J Invest Dermatol 2014;134:319-325.

27. Spagnolo F, Ghiorzo P, Orgiano L, et al. BRAF-mutant melanoma: treatment approaches, resistance mechanisms, and diagnostic strategies. Onco Targets Ther 2015;8:157-168.

28. Sumrall AL, Haggstrom DE, Burri SH, Giglio P. Use of NovoTTF ${ }^{\mathrm{TM}}-100 \mathrm{~A}$ in heavily pretreated patients with relapsed high grade gliomas, including previous or concurrent bevacizumab. Presented at the 18th Annual Meeting of the Society for Neuro-Oncology; November 21-24, 2013; San Francisco, California.

29. Stupp R, Wong ET, Kanner AA, et al. NovoTTF-100A versus physician's choice chemotherapy in recurrent glioblastoma: a randomised phase III trial of a novel treatment modality. Eur J Cancer 2012;48:2192-2202.

30. Stupp R, Taillibert S, Kanner A, et al. Tumor treating fields (TTFields): a novel treatment modality added to standard chemo- and radiotherapy in newly diagnosed glioblastoma-first report of the full dataset of the EF14 randomized phase III trial. [abstract] J Clin Oncol 2015;33(Suppl):Abstract 2000.

31. Kesari S, Ram Z. Tumor treating fields with chemotherapy compared to chemotherapy alone in glioblastoma patients at first recurrence: a post-hoc analysis of the EF-14 trial. Neuro Oncol 2015;17(Suppl 5):v14 